THE ASTROPHYSICAL JOURNAL, 531:665-675, 2000 March 10

(C) 2000. The American Astronomical Society. All rights reserved. Printed in U.S.A.

\title{
LUMINOSITY VERSUS PHASE-SPACE-DENSITY RELATION OF GALAXIES REVISITED
}

\author{
KeIICHI KodaIRA ${ }^{1}$ aND Nobunari KashiKaWA ${ }^{1}$ \\ National Astronomical Observatory of Japan, 2-21-1 Osawa, Mitaka, Tokyo, 181-8588 Japan \\ AND \\ TORU MisAwa ${ }^{2}$ \\ Department of Astronomy, School of Science, University of Tokyo, 7-3-1 Hongo, Bunkyo-ku, Tokyo, 113-0033 Japan \\ Received 1999 June 11 ; accepted 1999 November 26
}

\begin{abstract}
We reexamined the correlation between the $B_{T}$ magnitude and the phase-space-density parameter $w=\left(D_{25}^{2} v_{\mathrm{c}}\right)^{-1}$ of galaxies for the Virgo, the Coma, the Fornax, and the Perseus clusters in an effort to better understand the physical underpinning of the fundamental plane. A tight correlation $\left(B_{T}=a \log \right.$ $w+b$ ) common to different morphological types of galaxies (E, S0, S) was found for the Virgo and the Coma clusters, with $a=1.87 \pm 0.10$ and $1.33 \pm 0.11$, respectively. An investigation using only $\mathrm{E}$ galaxies was made for the four clusters. The results indicated that the empirical linear relation might be common among the Coma, the Fornax, and the Perseus clusters, with the Virgo Cluster showing deviation. This relation, which is another way to project the fundamental plane, has an expression insensitive to the morphology and may be suitable for treating galaxies of different morphological types collectively.

Subject headings: galaxies: clusters: general - galaxies: kinematics and dynamics galaxies: photometry - galaxies: structure
\end{abstract}

\section{INTRODUCTION}

Following the pioneering work by Brosche (1973), Watanabe, Kodaira, \& Okamura (1985) applied the principal component analysis to an appropriate set of surfacephotometric parameters of galaxies to identify significant independent variables that control the observed properties of galaxies of each morphological type. They found two principal components for $\mathrm{S}$ galaxies and one principal and one subordinate component for $\mathrm{E}$ galaxies. The twodimensional surface that is spun by the two corresponding eigenvectors forms a plane in a multidimensional space of the surface-photometric parameters of galaxies. This plane was presented as the diameter versus surface-brightness diagram (DSBD) collectively for E, S0, and S galaxies by Kodaira, Okamura, \& Watanabe (1983). More comprehensive analyses involving the spectroscopic parameters led to the concept of the fundamental plane (FP) for E galaxies by Dressler et al. (1987), Djorgovski \& Davis (1987), and Faber et al. (1987).

The linear relations that are produced by nearly edge-on projections of this kind of planes toward certain directions had been recognized as the Faber-Jackson relation for $\mathrm{E}$ galaxies (Faber \& Jackson 1976) and, similarly, as the Tully-Fisher relation for S galaxies (Tully \& Fisher 1977), which were widely utilized to yield distance estimates. The $D_{n}-\sigma$ relation for E galaxies (Dressler et al. 1987) was devised for the same application, based on the FP concept.

Another empirical relation was derived for $\mathrm{E}$ and $\mathrm{S}$ galaxies of the Virgo Cluster, by Kodaira (1989) as the luminosity versus phase-space-density (PSD) relation, not for the purpose of the distance estimate, but for better understanding of the physical meaning of the principal components defining galaxy properties. The PSD parameter was defined as $w \equiv\left(D^{2} v\right)^{-1}$, where $D$ was the photo-

\footnotetext{
${ }^{1}$ keiichi.kodaira@nao.ac.jp, kashik@zone.mtk.nao.ac.jp.

2 misawatr@cc.nao.ac.jp.
}

metric diameter and $v$ was the central velocity dispersion $\left(\sigma_{0}\right)$ for E galaxies or the rotation velocity represented by the $\mathrm{H}_{\mathrm{I}}$ line width $\left(W_{20}\right)$ for $\mathrm{S}$ galaxies. Since quantity $\left(\mathrm{G} D^{2} v\right)^{-1}$ has a dimension of PSD of a single-particle ensemble in the virial equilibrium, $w$ may represent a sort of an average PSD of a galaxy as a stellar ensemble.

Bender, Burstein, \& Faber (1992) introduced the concept of the $\kappa$-space for E and S0 galaxies, a three-dimensional space that had coordinates along mass parameter $\left(\kappa_{1}\right)$, mass to luminosity ratio parameter $\left(\kappa_{3}\right)$, and $I_{\mathrm{e}}^{3} \times M / L$ parameter $\left(\kappa_{2}\right)$, where $I_{\mathrm{e}}$ was the average surface brightness within the equivalent radius, $r_{\mathrm{e}}$. The plane $\left(\kappa_{1}, \kappa_{2}\right)$ in this $\kappa$-space was defined by the distribution of dynamically hot galaxies (Es and a part of S0s) in the Virgo Cluster and is close to being a face-on view of FP. Its nearly edge-on projection along the $\kappa_{2}$ axis was found to be $\kappa_{3}=0.15 \kappa_{1}+0.36$, which was interpreted in the simple context of $L_{B}=M \times\left(L_{B} / M\right)$, with $M$ being a kind of virial mass $\left(\mathrm{G}^{-1} r_{\mathrm{e}} \sigma_{0}^{2}\right)$ under an inference of $M / L_{B} \propto M^{0.15}$. They suggested that the dynamically hot galaxies such as E and S0 might have been formed by dissipationless merging in keeping approximate relation $L \propto M$. This framework of the $\kappa$-space was later applied by Burstein et al. (1997) to $S$ galaxies and, further, to other stellar systems such as groups and clusters of galaxies, and globular clusters (see also Djorgovski 1995).

As for S galaxies specifically, Chiba \& Yoshii (1995) proposed a relation for disk galaxies to yield distance estimates by using the correlation of the radial scale length of the disk $\left(r_{\mathrm{d}}\right)$ with the specific combination of the central surface brightness $\left(I_{0}\right)$ and the rotation velocity $(v) ; r_{\mathrm{d}} \propto\left(v^{2} I_{0}^{-1}\right)^{p}$ with $p \sim 0.5$. Koda \& Sofue (2000) have reported that they have found the plane equivalent to FP for S galaxies, which may degenerate to the luminosity versus specific angular momentum relation with the specific-angular-momentum parameter being $D v$.

In the present paper we will reinvestigate the luminosity versus PSD relation by extending the sample to S0s in the Virgo Cluster, and to E, S0, and S galaxies in the Coma 
Cluster, and finally to E galaxies in the Fornax and the Perseus clusters. This study is undertaken in an effort further to elucidate the physical nature of the interrelation, rather than to develop an operating method for getting the distances of galaxies. The findings are discussed in $\S 3$ in connection to FP and the structure of the multidimensional space for galaxy parameters.

\section{2. $B_{T}$ MAGNITUDE VERSUS PHASE-SPACE-DENSITY PARAMETER RELATION}

In the present study we adopt the total $B$ magnitude, $B_{T}$, as the luminosity parameter, the diameter at $\mu_{B}=25 \mathrm{mag}$ $\operatorname{arcsec}^{-2}, D_{25}$ in units of $1^{\prime \prime}$, as the size parameter, and the axial ratio of $S$ galaxies at $\mu_{B}=25 \mathrm{mag} \operatorname{arcsec}^{-2}, R_{25} \equiv b / a$, in place of the parameters defined in the $V$ band, $V_{26}, D_{26}$, and $R_{26}$ in Kodaira (1989). The adopted velocity data are the central velocity dispersion, $\sigma_{0}$, for $\mathrm{E}$ and $\mathrm{S} 0$ galaxies, and the $\mathrm{H}_{\mathrm{I}} 21 \mathrm{~cm}$ line width at $20 \%$ level, $W_{20}$, for $\mathrm{S}$ galaxies. As for $\mathrm{S}$ galaxies, the observational data, in particular the velocity data, are subject to the inclination effects, and the present $\mathrm{S}$ sample is limited to those of $30^{\circ} \leq i \leq 65^{\circ}$ in order to minimize the uncertainties in the inclination corrections, where $i$ is to be derived through the conventional formula using $R_{25}$ from de Vaucouleurs et al. 1991 (hereafter RC3) as adopted in Kodaira \& Watanabe (1988). The inclination-corrected velocity for $\mathrm{S}$ galaxies is defined as $0.5 W_{20} / \sin i$. For simplicity, no higher order corrections to other observational data, including those for the inisotropy of $\sigma_{0}$ and the triaxiality for $\mathrm{E}$ and $\mathrm{S} 0$ galaxies, are applied. As the velocity parameter to define the phasespace-density parameter, $w$, we adopt the circular velocity, $v_{\mathrm{c}} \equiv \sqrt{3} \sigma_{0} / 1.1$ or $v_{\mathrm{c}} \equiv 0.5 W_{20} / \sin i$ in units of $\mathrm{km} \mathrm{s}^{-1}$, which corresponds to the flat rotation velocity in the gravitational potential of an isothermal dark halo of a galaxy (see Shimasaku 1993). The additional factor of $\sqrt{3} / 1.1=$ 1.57 introduced here corresponds to the factor of about 1.4 or $\sqrt{2}$, which was found empirically by Whitmore $\&$ Kirshner (1981) and derived theoretically by Binney \& Tremaine (1987). Kodaira (1989) also derived the same factor to convert $\sigma_{0}$ into $0.5 W_{20} / \sin i$ for the disk galaxies of the earliest morphological types.

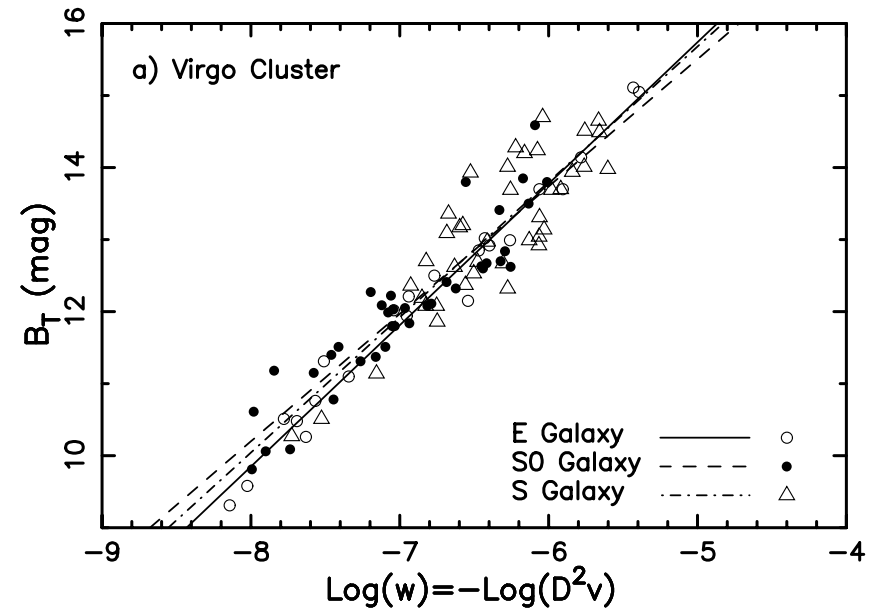

The least-square fitting of a regression line in a form of $B_{\mathrm{T}}=a \log w+b$ with $w \equiv\left(D_{25}^{2} v_{\mathrm{c}}\right)^{-1}$ will be carried out separately for $\mathrm{E}(T \leq-3)$, $\mathrm{S} 0(-2 \leq T \leq 0)$, and $\mathrm{S}(T \geq 1)$ galaxies. Note that no corrections due to the difference in the distance of individual galaxies in each cluster are applied to $B_{T}$ or $D_{25}$; the distance difference within a cluster is regarded as negligible compared to the cluster distance. Thus we are using $B_{T}$ as a luminosity parameter. The sample galaxies are listed in Appendix A with their data using the numbers in catalogs $(\mathrm{N}=\mathrm{NGC}, \mathrm{I}=\mathrm{IC}$, $\mathrm{U}=\mathrm{UGC}$, and $\mathrm{Z}=\mathrm{Z}$ wickey Catalog). Since the photometric and velocity data are adopted from various sources, their systematic trends are investigated in Appendix B. Significant ones should be taken into account in comparing the results of the correlation analyses.

\subsection{Virgo Cluster}

Kodaira (1989) carried out the regression-line analysis for $\mathrm{E}$ and S galaxies in the Virgo Cluster, while S0 galaxies are included in the present study. The sample galaxies and their data are given in Appendix A (Tables 4-6). The data have been adopted from Binggeli, Sandage, \& Tammann (1985) $\left(T, B_{T}, D_{25}\right)$, Davoust, Paturel, \& Vauglin (1985) $\left(\sigma_{0}\right)$, and RC3 $\left(W_{20}\right)$. The resulting regression lines are shown in Figure $1 a$, and their parameters are given in Table 1 with the number of sample galaxies $(n)$ and the correlation coefficient. We find tight correlation also for S0 galaxies and notice that by adopting the circular velocity, $v_{\mathrm{c}}$, the regression lines almost coincide each other among the three types of galaxies. We note that the values of $a$ for $\mathrm{E}$ and $\mathrm{S}$ galaxies in the present study are different from those in Kodaira

TABLE 1

REgRESSION LiNe ANALYSES $B_{T}=a \log w+b$ FOR THE VIRGO CluSTER

\begin{tabular}{ccccc}
\hline \hline Type & $a$ & $b$ & $n$ & Correlation Coefficient \\
\hline E ....... & 1.964 & 25.56 & 22 & 0.9886 \\
S0 ...... & 1.769 & 24.35 & 37 & 0.9234 \\
S ...... & 1.879 & 25.07 & 40 & 0.8630 \\
\hline
\end{tabular}

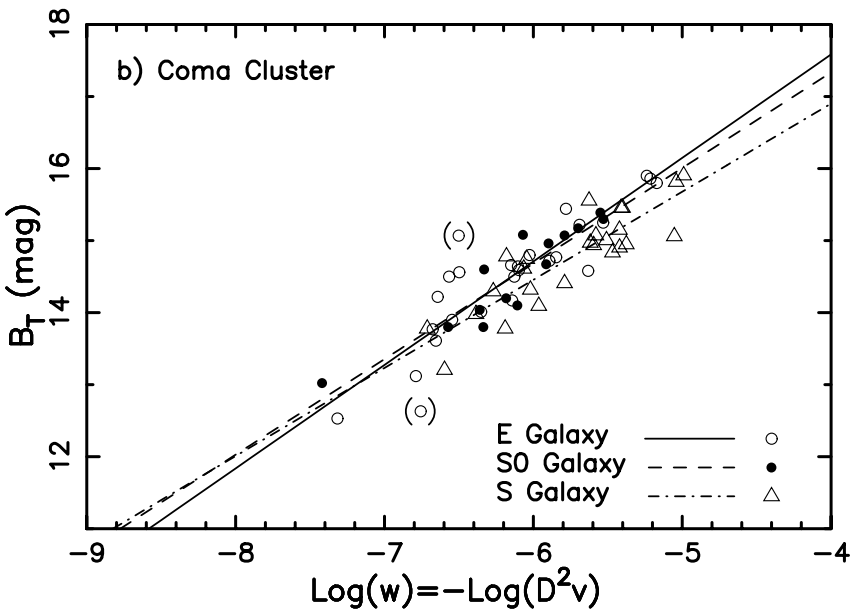

FIG. 1. $-B_{T}$ magnitude vs. phase-space-density $(w)$ relation. $w=\left(D_{25}^{2} v_{\mathrm{c}}\right)^{-1}$, with $v_{\mathrm{c}}=\sqrt{3} \sigma_{0} / 1.1$ for E and S0, and $v_{\mathrm{c}}=0.5 W_{20} /$ sin $i$ for $\mathrm{S}$ galaxies. The coefficients of the regression lines are given in Tables 1,2, and 3. The E regression lines for the Coma Cluster are for the case including the two deviating galaxies, which are marked by parentheses (see text). (a) Virgo Cluster $(b)$ Coma Cluster: The data for S galaxies are plotted after the $B_{T}$ transformation from Fukugita et al. (1991) to the RC3 system, based on the analysis in Appendix B. (c) E galaxies of the four clusters in the sample of Dressler et al. (1987): The regression lines are shifted corresponding to the distance of the Coma Cluster according to the distances, $R$, in Table 3. 


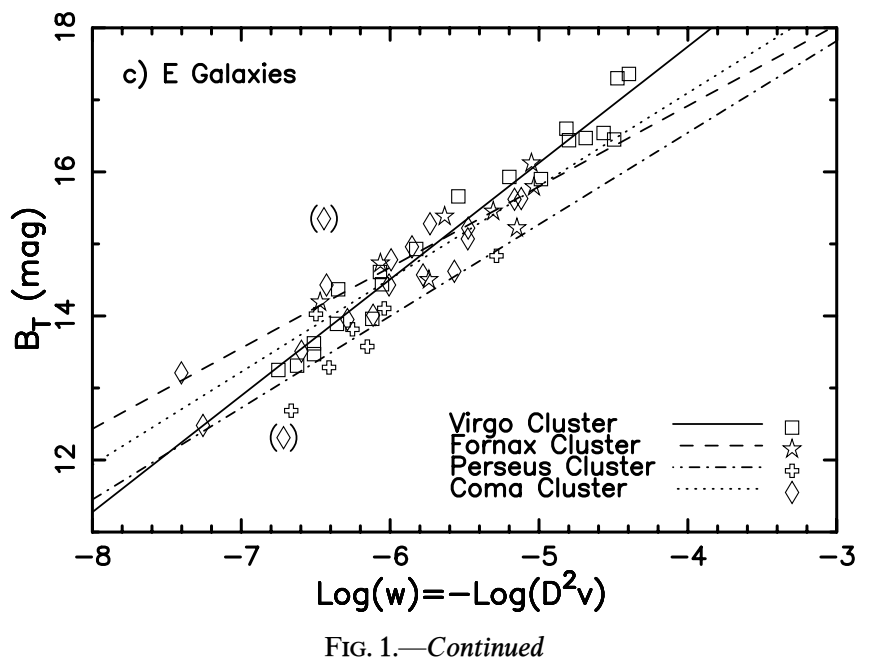

(1989) ( $a=1.50$ for E, 2.24 for S), largely because of the difference in the adopted photometric band.

By applying the Tully-Fisher relation, Yasuda, Fukugita, \& Okamura (1997) suggested that $\mathrm{S}$ galaxies in the Virgo Cluster are distributed in a substantially extended region along the line of sight from 12 to $30 \mathrm{Mpc}$. Therefore, the present results, particularly for the Virgo $S$ galaxies, need to be taken with caution although the $B_{T}$ versus $\log w$ relation is rather insensitive to distance errors. We note that the faint $\mathrm{S}$ and $\mathrm{S} 0$ galaxies $\left(B_{T} \gtrsim 13.5\right)$ show excess in the domain above the regression lines in Figure $1 a$. This may be partly due to the low accuracy of the photometric data for faint, small galaxies.

\subsection{Coma Cluster}

The sample galaxies for the Coma Cluster and their data are given in Appendix A (Tables 7-9), which have been adopted from RC3 ( $T$ for $\mathrm{S}, B_{T}$ for $\mathrm{E}$ and S0, $D_{25}$ ), Fukugita et al. (1991) $\left(B_{T}\right.$ and $W_{20}$ for S), and Scodeggio, Giovanelli, \& Haynes (1998a, 1998b) ( $T$ and $\sigma_{0}$ for $\mathrm{E}$ and S0). The stellar dispersion, $\sigma_{s}$, given in Scodeggio et al. (1998a, $1998 \mathrm{~b}$ ) was transformed into $\sigma_{0}$ as $\sigma_{0} \equiv 1.1 \sigma_{s}$ according to their empirical calibration. The $\mathrm{S}$ sample was adopted from Fukugita et al. (1991) who did not classify the subtypes. The type $T$ from RC3 are not available for all of the adopted sample. The present $S$ sample includes a galaxy of $T=-2$ (Z160067), which, however, does not show any clear deviation among the sample. The resulting regression lines are shown in Figure $1 b$, and the fitting parameters are given in Table 2.

TABLE 2

Regression Line ANALYSES $B_{T}=a \log w+b$ FOR THE COMa Cluster

\begin{tabular}{ccccc}
\hline \hline Type & $a$ & $b$ & $n$ & Correlation Coefficient \\
\hline E $\ldots \ldots .$. & 1.437 & 23.33 & 26 & 0.8844 \\
& 1.404 & 23.13 & 24 & 0.9309 \\
S $0 \ldots \ldots$ & 1.327 & 22.64 & 14 & 0.9217 \\
S $\ldots \ldots .$. & 1.223 & 21.79 & 25 & 0.8755 \\
\hline
\end{tabular}

NotE.-The data for $\mathrm{E}$ in italics are for the regression-line fitting without the two galaxies showing large deviation in Fig. $1 b$. The data for $\mathbf{S}$ are for the regression-line fitting after the transformation of $B_{T}$ from the Fukugita et al. 1991 system to the RC3 system.
In Figure $1 b$ we notice that there are two E galaxies that show almost $3 \sigma$ deviations (N4874, N4872). The brighter one, N4874, is a CD galaxy ( $T=-4$ in RC3) west to the other CD galaxy N4889 in the central part of the Coma Cluster, while the fainter one, $\mathrm{N} 4872(T=-2$ in RC3), is located in the halo of N4874 with a few of other galaxies. The cause of the deviations might be a kind of photometric confusion. The fitting parameters for the E sample excluding these two deviators are given in Table 2. The regression line in Figure $1 b$ is for the case including the two deviating galaxies. Since $B_{T}$ in Fukugita et al. (1991) shows significant systematic deviation from that in RC3 (see Appendix B), the results for $\mathrm{S}$ galaxies that have been transformed into the RC3 system are given in Table 2 and are plotted in Fig. $1 b$ ).

We find again common distribution among E, S0, and S galaxies. The gradient of the regression line is $a=1.33 \pm 0.11$ for the Coma Cluster, which is different from $a=1.87 \pm 0.10$ for the Virgo Cluster. There seems to be a fine difference in the value of coefficient, $a$, among different types of galaxies in a cluster, indicating a tendency of $a(\mathrm{E}) \gtrsim a(\mathrm{~S} 0) \gtrsim a(\mathrm{~S})$, when the results for the Virgo S galaxies are taken into account with a lower weight.

\subsection{E Galaxies of the Virgo, Coma, Fornax, and Perseus Clusters}

In this section we confine our sample to $E$ galaxies in the Fornax and the Perseus clusters, in addition to the Virgo and the Coma clusters. Their data are adopted from Dressler et al. (1987), except for $D_{25}$, which are adopted from RC3. The adopted samples and their data are given in

TABLE 3

Regression Line ANALYSes $B_{T}=a \log w+b$ FOR THE E GalaXIES OF the Four Clusters IN Dressler ET AL. 1987

\begin{tabular}{lcrrcc}
\hline \hline Cluster & $a$ & $b$ & $n$ & $\begin{array}{c}\text { Correlation } \\
\text { Coefficient }\end{array}$ & $\begin{array}{c}R \\
\left(\mathrm{~km} \mathrm{~s}^{-1}\right)\end{array}$ \\
\hline Virgo & 1.616 & 24.20 & 20 & 0.9827 & 1333 \\
Fornax & 1.121 & 21.40 & 8 & 0.8879 & 1422 \\
Perseus & 1.273 & 21.64 & 7 & 0.8443 & 6050 \\
Coma & 1.291 & 22.26 & 18 & 0.8371 & 7461 \\
& 1.246 & 21.98 & 16 & 0.9359 & \\
\hline
\end{tabular}

Note.-The data for the Coma cluster in italic are for the regression-line fitting without the two galaxies showing large deviation in Fig. 1 c. $R$ is the distance expressed as recession velocity in Faber et al. 1989. 
Appendix A (Table 10), and the regression lines are shown in Figure $1 c$, with the fitting parameters given in Table 3. The resulting regression lines for $\mathrm{E}$ galaxies of the Virgo and the Coma clusters in the present subsection are different from those obtained in $\S \S 2.1$ and 2.2. These differences are mostly caused by the fact that the samples and the data in this subsection are different from those adopted in the preceding subsections (see Appendices A and B). In the case of the Coma Cluster, the parameters for the fitting without the two deviating galaxies are indicated in italic in Table 3 . The regression line for the Coma Cluster in Figure $1 c$ is for the case including the two deviating galaxies. The gradient of the regression line is similar among the sample clusters $(a=1.21 \pm 0.09)$ with deviation of the Virgo Cluster $(a=1.62)$.

For convenience of comparison, the plots for the different clusters are overlapped in Figure $1 c$ by applying the distance correction to each cluster relative to the Coma corresponding to the distance, $R$, in Table 3 , which were given by Faber et al. (1989) based on the $D_{n}-\sigma$ relation. We notice that the Perseus galaxies appear to deviate systematically from the others toward the lower side in Figure 1c. Another distance scale such as was proposed by Jerjen \& Tammann (1993) or by Hudson et al. (1997) leads to a difference of the distance modulus between the Coma and the Perseus clusters $\Delta(m-M)=0.57$ or 0.72 , respectively, in contrast to $\Delta(m-M)=0.45$ in Table 3, improving the overlapping of the galaxies of the two clusters in Figure 1c.

We also notice that the upward deviator on the bright end in Figure $1 c$ is the third brightest galaxy in the present sample of the Coma Cluster in Table 10, N4839. This galaxy is classified as S0 in Table 8 and is located at the center of the southwest subcluster. Recent X-ray observation from $A S C A$ (see Watanabe et al. 1999) has revealed that this subcluster may have a separate halo structure from the main halo of the Coma Cluster. The deviation of N4839 in Figure $1 c$ might be related to this fact.

\section{SUMMARY AND DISCUSSION}

Although the analyzed sample is limited, we may draw some inferences as follows.

For the Virgo and the Coma clusters:

1. The $B_{T}$ versus $\log w$ relation $\left(B_{\mathrm{T}}=a \log w+b\right)$ is valid for a wide range of galaxy types, E, S0, and S. The coefficient $a$ appears to show a tendency of $a(\mathrm{E}) \gtrsim a(\mathrm{~S} 0) \gtrsim$ $a(\mathbf{S})$.

For E galaxies of the Virgo, the Fornax, the Perseus, and the Coma clusters:

2. The coefficient $a$ is almost common among the clusters, with the Virgo Cluster showing deviation.

In addition we note as follows:

3. The coefficients of the regression lines are depending upon the data sources as Appendix B indicates. One must be careful in discussing the physical meaning of the empirical relations using the nominal values of the fitting coefficients.

4. The values of the coefficients of the regression lines depend upon the adopted photometric band. Some of the apparent properties of the empirical relations as are pointed out above may be subject to modification if photometric bands other than the $B$ are applied.
In summary, we have found that the $B_{T}$ versus $\log w$ relation can be regarded as a tight empirical relation that is almost common to a wide range of galaxy types in each cluster. The relation might be common for majority of nearby clusters, though the Virgo Cluster may show possible deviation. Since the Virgo Cluster is the nearest among the sample clusters and is suspected to have a widespread complicated structure, further studies are needed to confirm its actual deviation.

The relation, $B_{T}=a \log w+b$, is one of the nearly edge-on projections of FP and almost equivalent to the relation $\kappa_{3}=\alpha \kappa_{1}+\beta$ in the $\kappa$-space of Bender et al. (1992), as is shown below. Both can be transformed into the relation in which the effective radius $\left(r_{e}\right)$ is expressed as a function of distance-independent parameters, the velocity $(v)$, and the mean surface brightness within $r_{e}\left(I_{e}\right)$. The $\kappa$ relation is rewritten by the definition of $\kappa_{1}$ and $\kappa_{3}$ as

$$
\log r_{e}=\gamma \log v+\delta \log I_{e}+\text { const., }
$$

with $\gamma \equiv 2(2-\sqrt{6} \alpha) /(2+\sqrt{6} \alpha)$ and $\delta \equiv-2 /(2+\sqrt{6} \alpha)$, while the $B_{T}$ versus $\log w$ relation is transformed into

$$
\begin{aligned}
\log r_{e}= & p \log v+q \log I_{e}+p \log \left\{f^{-1}\left(A I_{25} / I_{e}\right)\right\} \\
& + \text { const. }
\end{aligned}
$$

with $p \equiv a /(5-2 a), q \equiv 2.5 /(2 a-5)$, and $I_{25}$ being the surface brightness corresponding to $\mu=25 \mathrm{mag} \operatorname{arcsec}^{-2}$. In deriving equation (2), we have assumed that the surfacebrightness profile of a galaxy along the major axis is a monotonous function, $I(r)=I_{0} f\left(r / r_{0}\right)$, with $I_{0}$ and $r_{0}$ being the representative surface brightness and radial scale; $f^{-1}\left(I_{25} A / I_{e}\right)=r_{25} / r_{0}$ with definition of $A \equiv I_{e} / I_{0}$. If we stipulate $\gamma=p$, the difference $\Delta$ between the right terms of the two expressions (1) and (2) becomes

$$
\Delta \equiv p\left[-0.75 \log I_{0}+\log \left\{f^{-1}\left(I_{25} / I_{0}\right)\right\}\right]+\text { const. }
$$

with $p$ being the same coefficient as in equation (2) and of $\mathrm{O}(1)$ for the range of $a$ obtained in the present paper. As the value of $\alpha$ is 0.15 for $E$ galaxies and larger than this for $S$ galaxies (say 0.30) (see Burstein et al. 1997), the condition $\gamma=p$ leads to $a=1.83$ for $\mathrm{E}$ and a value slightly smaller than that for $\mathrm{S}(1.60)$, which are comparable to the $a$ values found in the present paper. The term in the square brackets in equation (3) varies monotonously and its absolute value remains almost constant at $0.93 \pm 0.23$ for $18.5 \lesssim \mu_{0, B} \lesssim$ 22.5 in the case of $f(x)=\exp \left\{-x^{1 / 4}\right\}$, and at $0.60 \pm 0.30$ for $19.5 \lesssim \mu_{0, B} \lesssim 23.5$ in the case of $f(x)=\exp \{-x\}$. These ranges of $\mu_{0, B}$ for $\mathrm{E}$ and $\mathrm{S}$ galaxies are estimated from the $V$-band surface photometry (Kodaira, Watanabe, \& Okamura 1986; Kodaira et al. 1990) by assuming $B-V=1.0$ and 0.6 for $\mathrm{E}$ and $\mathrm{S}$, respectively. In conclusion, the $B_{T}$ versus $\log w$ relation is almost equivalent to the $\kappa_{1}$ versus $\kappa_{3}$ relation as a nearly edge-on projection of FP and has an expression insensitive to the difference of the morphological type of galaxies. The concept of PSD, therefore, may be suitable for collective study of galaxies in connection to FP.

When we regard a galaxy to be a relaxed dynamical ensemble of stellar particles imbedded in a relaxed dark halo, the global properties of the galaxy and the dark halo may well be characterized by their mass and the average PSD. The physical process involved in the galaxy formation and evolution such as mass loss, merging, or dissipation 
may ideally be investigated on the mass versus average PSD plane. In practice, however, we directly observe not the mass but the luminosity for stars, and indirectly estimate the effective virial mass including both stars and dark halo. The observed luminosity in a certain photometric band, $L_{\lambda}$, is related to the stellar mass, $m_{s}$, with a mass/luminosity ratio $\left(m_{\mathrm{s}} / L_{\lambda}\right)=\left(m_{\mathrm{s}} / L\right)\left(L / L_{\lambda}\right)$, which is generally dependent on the evolution of galaxies. As we are mainly concerned with normal and giant galaxies at the present epoch, $m_{s}$ approximately represents the barion mass of a galaxy. When we take the total mass, including the mass of the dark halo, $m_{T}$, into account, we have to consider a relation $L_{\lambda}=$ $m_{\mathrm{T}}\left(m_{\mathrm{s}} / m_{\mathrm{T}}\right)\left(L / m_{\mathrm{s}}\right)\left(L_{\lambda} / L\right)$. When we study galaxies collectively on a mass versus PSD plane, we need more detailed consideration about the factor $m_{s} / m_{\mathrm{T}}$, which may be significantly affected by the formation and the initial-phase evolution of galaxies.

The mass $m_{\mathrm{T}}$ and PSD of the total system are better investigated with help of X-ray data. Fritsch \& Buchert (1999) introduced a concept of FP for clusters of galaxies using the optical and X-ray data. The involved parameters are the total $B$-band luminosity $L_{\mathrm{O}}$, the total X-ray luminosity $L_{\mathrm{X}}$, and the half-light radii $R_{\mathrm{O}}$ (optical) and $R_{\mathrm{X}}(\mathrm{X}$ ray) of each cluster. Their nearly edge-on projection of the cluster FP is represented by relation $\log L_{\mathrm{O}}=0.84 \log R_{\mathrm{O}}$ $+0.21 \log L_{\mathrm{X}}+$ const., and the deviation from the relation is suggested to be an indication of the unrelaxed dynamical system. If there are differences in the large-scale structure of the dark halo embedding a cluster of galaxies, they may have consequences upon the local structure of the dark halo of each member galaxies, leading to differences in the $B_{T}$ versus $\log w$ relation among different clusters, such as is suspected for the Virgo Cluster. The results of the present study indicate that the relaxed clusters may show the common $B_{T}$ versus $\log w$ relation. It is desirable to include also the optical spectroscopic parameters in this kind of cluster studies. Using future X-ray data of higher resolution, we may do similar studies of individual galaxies, involving both optical and X-ray data to better understand the physical underpinning of the galaxy FP.

The authors acknowledge that Kazuhiro Shimasaku and Naoki Yasuda kindly made their observational data available to the present analyses. They wish to thank anonymous referee for his constructive advice to clarify the purpose of this paper and to present the mathematical connection of the PSD relation to the FP concept.

\section{APPENDIX A}

\section{DATA LIST}

The sample galaxies and their data for the present regression-line analyses are summarized in the following Tables 4-10.

TABLE 4

Virgo Cluster E GalaXies

\begin{tabular}{|c|c|c|c|c|c|}
\hline Galaxy & $T$ & $B_{T}$ & $\log D_{25}$ & $\log v_{\mathrm{c}}$ & $-\log w$ \\
\hline N4168 $\ldots$. & -5 & 12.21 & 2.23 & 2.48 & 6.94 \\
\hline N4239 .... & -5 & 13.70 & 2.03 & 2.01 & 6.06 \\
\hline N4261 ................ & -5 & 11.31 & 2.37 & 2.77 & 7.51 \\
\hline N4318 ............... & -5 & 14.14 & 1.79 & 2.21 & 5.78 \\
\hline N4365 ............... & -5 & 10.51 & 2.57 & 2.64 & 7.78 \\
\hline N4374 ............... & -5 & 10.26 & 2.48 & 2.67 & 7.63 \\
\hline N4387 ...... & -5 & 13.02 & 2.05 & 2.33 & 6.43 \\
\hline N4434 ............... & -5 & 12.99 & 1.98 & 2.30 & 6.26 \\
\hline N4458 .............. & -5 & 12.92 & 2.08 & 2.24 & 6.40 \\
\hline N4464 ............... & -5 & 13.70 & 1.84 & 2.23 & 5.90 \\
\hline N4467 ............... & -5 & 15.05 & 1.63 & 2.14 & 5.39 \\
\hline N4472 ................ & -5 & 9.31 & 2.73 & 2.69 & 8.14 \\
\hline N4473 ............... & -5 & 11.10 & 2.43 & 2.49 & 7.34 \\
\hline N4478 ............... & -5 & 12.15 & 2.09 & 2.37 & 6.54 \\
\hline 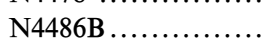 & -5 & 15.11 & 1.46 & 2.52 & 5.43 \\
\hline N4486 ............... & -5 & 9.58 & 2.64 & 2.75 & 8.02 \\
\hline N4550 ............... & -5 & 12.50 & 2.32 & 2.13 & 6.77 \\
\hline N4551 .............. & -5 & 12.85 & 2.08 & 2.31 & 6.47 \\
\hline N4564 ............... & -5 & 12.02 & 2.27 & 2.50 & 7.04 \\
\hline N4621 .............. & -5 & 10.76 & 2.49 & 2.59 & 7.57 \\
\hline N4636 .............. & -5 & 10.48 & 2.57 & 2.56 & 7.69 \\
\hline N4660 ................ & -5 & 11.94 & 2.22 & 2.52 & 6.95 \\
\hline
\end{tabular}


TABLE 5

VIRGo Cluster S0 GalaXies

\begin{tabular}{|c|c|c|c|c|c|}
\hline Galaxy & $T$ & $B_{T}$ & $\log D_{25}$ & $\log v_{\mathrm{c}}$ & $-\log w$ \\
\hline $\mathrm{N} 4200 \ldots$ & -2 & 13.85 & 2.03 & 2.12 & 6.17 \\
\hline N4259 .............. & -2 & 14.59 & 1.84 & 2.42 & 6.09 \\
\hline N4262 .............. & -2 & 12.41 & 2.12 & 2.45 & 6.69 \\
\hline N4267 .............. & -2 & 11.80 & 2.32 & 2.41 & 7.05 \\
\hline N4281 ............... & -2 & 12.27 & 2.27 & 2.66 & 7.20 \\
\hline N4292 ............... & 0 & 13.50 & 2.10 & 1.94 & 6.13 \\
\hline N4296 ............. & -2 & 13.80 & 2.03 & 2.50 & 6.56 \\
\hline N4324 .............. & -1 & 12.60 & 2.17 & 2.11 & 6.44 \\
\hline N4339 ............... & -2 & 12.32 & 2.15 & 2.33 & 6.62 \\
\hline N4340 ............... & -1 & 12.03 & 2.39 & 2.27 & 7.04 \\
\hline $\mathrm{N} 4350 \ldots \ldots \ldots \ldots \ldots$ & -2 & 11.99 & 2.28 & 2.52 & 7.08 \\
\hline N4371 ............... & -2 & 11.80 & 2.37 & 2.30 & 7.03 \\
\hline N4377 ............... & -2 & 12.67 & 2.04 & 2.34 & 6.42 \\
\hline N4379 .............. & -2 & 12.62 & 2.10 & 2.06 & 6.26 \\
\hline N4382 ............... & -2 & 10.09 & 2.63 & 2.48 & 7.74 \\
\hline N4406 ............... & -2 & 10.06 & 2.65 & 2.60 & 7.90 \\
\hline N4417 ............... & -2 & 12.08 & 2.34 & 2.14 & 6.81 \\
\hline N4429 ............... & -2 & 11.15 & 2.52 & 2.54 & 7.58 \\
\hline N4435 ............... & -2 & 11.84 & 2.25 & 2.44 & 6.93 \\
\hline N4442 .............. & -2 & 11.40 & 2.44 & 2.58 & 7.46 \\
\hline N4459 ............... & -2 & 11.37 & 2.36 & 2.45 & 7.16 \\
\hline N4461 ............... & -1 & 12.09 & 2.35 & 2.43 & 7.12 \\
\hline N4468 .............. & 0 & 13.80 & 1.96 & 2.10 & 6.01 \\
\hline 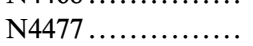 & -2 & 11.31 & 2.38 & 2.51 & 7.26 \\
\hline N4489 ............... & -2 & 12.84 & 2.12 & 2.06 & 6.29 \\
\hline N4526 .............. & -2 & 10.61 & 2.64 & 2.71 & 7.98 \\
\hline $\mathrm{N} 4528 \ldots \ldots \ldots \ldots$ & -2 & 12.70 & 2.04 & 2.25 & 6.32 \\
\hline N4552 .............. & -2 & 10.78 & 2.40 & 2.65 & 7.45 \\
\hline N4578 ............... & -2 & 12.22 & 2.34 & 2.38 & 7.06 \\
\hline $\mathrm{N} 4596 \ldots \ldots \ldots \ldots \ldots$ & -1 & 11.51 & 2.37 & 2.36 & 7.10 \\
\hline N4598 ............... & -2 & 13.41 & 2.08 & 2.18 & 6.33 \\
\hline N4608 ............... & -2 & 12.05 & 2.28 & 2.41 & 6.97 \\
\hline N4638 .............. & -2 & 12.11 & 2.23 & 2.33 & 6.79 \\
\hline N4649 ............... & -2 & 9.81 & 2.64 & 2.72 & 7.99 \\
\hline N4733 ............. & -2 & 12.63 & 2.15 & 2.16 & 6.45 \\
\hline N4754 $\ldots \ldots \ldots \ldots \ldots$ & -2 & 11.51 & 2.45 & 2.52 & 7.41 \\
\hline N4762 .............. & -2 & 11.18 & 2.72 & 2.41 & 7.85 \\
\hline
\end{tabular}


TABLE 6

VIrgo Cluster S GalaXies

\begin{tabular}{|c|c|c|c|c|c|c|}
\hline Galaxy & $T$ & $B_{T}$ & $\log D_{25}$ & $R_{25}$ & $\log v_{\mathrm{c}}$ & $-\log w$ \\
\hline I3021 ............. & 1 & 14.70 & 2.04 & 0.62 & 1.96 & 6.04 \\
\hline I3033 ............. & 5 & 14.65 & 1.88 & 0.66 & 1.91 & 5.66 \\
\hline I768 ............. & 5 & 14.28 & 2.00 & 0.50 & 2.23 & 6.22 \\
\hline N4165 .............. & 1 & 14.20 & 1.96 & 0.66 & 2.25 & 6.16 \\
\hline I769 .............. & 3 & 13.17 & 2.18 & 0.68 & 2.24 & 6.60 \\
\hline N4193 .............. & 5 & 13.20 & 2.14 & 0.51 & 2.30 & 6.58 \\
\hline $\mathrm{N} 4212 \ldots \ldots \ldots \ldots \ldots$ & 5 & 11.86 & 2.26 & 0.62 & 2.23 & 6.75 \\
\hline N4237 ............ & 5 & 12.53 & 2.14 & 0.65 & 2.23 & 6.50 \\
\hline N4246 ............ & 5 & 13.36 & 2.18 & 0.54 & 2.32 & 6.67 \\
\hline $\mathrm{I} 776 \ldots \ldots \ldots$ & 6 & 14.01 & 2.11 & 0.60 & 2.06 & 6.28 \\
\hline N4260 ............. & 1 & 12.70 & 2.20 & 0.50 & 2.43 & 6.82 \\
\hline N4273 ............. & 5 & 12.37 & 2.14 & 0.65 & 2.28 & 6.56 \\
\hline N4298 .............. & 5 & 12.08 & 2.28 & 0.56 & 2.20 & 6.75 \\
\hline N4334 .............. & 2 & 13.93 & 2.16 & 0.48 & 2.21 & 6.53 \\
\hline I3259 ............. & 5 & 14.24 & 2.03 & 0.55 & 2.02 & 6.08 \\
\hline N4353 ............. & 5 & 13.94 & 1.89 & 0.63 & 2.07 & 5.84 \\
\hline N4351 .............. & 5 & 13.04 & 2.09 & 0.68 & 1.89 & 6.06 \\
\hline N4370 ............. & 1 & 13.69 & 1.99 & 0.52 & 2.28 & 6.26 \\
\hline N4376 ............ & 6 & 13.69 & 2.00 & 0.62 & 1.98 & 5.98 \\
\hline N4380 ............ & 2 & 12.36 & 2.35 & 0.55 & 2.23 & 6.93 \\
\hline N4405 ............. & 5 & 12.99 & 2.08 & 0.65 & 1.97 & 6.13 \\
\hline N4413 ............. & 4 & 12.97 & 2.17 & 0.65 & 2.08 & 6.41 \\
\hline I3356 ............. & 9 & 14.49 & 2.00 & 0.59 & 1.66 & 5.69 \\
\hline N4420 ............ & 5 & 12.67 & 2.13 & 0.48 & 2.05 & 6.31 \\
\hline N4424 .............. & 1 & 12.32 & 2.35 & 0.50 & 1.58 & 6.28 \\
\hline N4451 .............. & 5 & 13.31 & 1.95 & 0.65 & 2.17 & 6.06 \\
\hline I3414 ............. & 5 & 13.70 & 2.01 & 0.60 & 1.90 & 5.92 \\
\hline N4480 ............ & 3 & 13.09 & 2.20 & 0.51 & 2.29 & 6.68 \\
\hline N4498 ............ & 5 & 12.62 & 2.29 & 0.54 & 2.06 & 6.63 \\
\hline I797 .............. & 5 & 14.01 & 1.88 & 0.69 & 2.00 & 5.76 \\
\hline N4501 .............. & 4 & 10.27 & 2.62 & 0.54 & 2.49 & 7.73 \\
\hline N4535 ............ & 5 & 10.51 & 2.61 & 0.71 & 2.31 & 7.53 \\
\hline I3517 ............. & 7 & 14.51 & 1.97 & 0.63 & 1.82 & 5.79 \\
\hline I3521 ............. & 9 & 13.98 & 1.85 & 0.69 & 1.91 & 5.63 \\
\hline N4567 ............ & 5 & 12.08 & 2.25 & 0.68 & 2.33 & 6.83 \\
\hline N4595 ............. & 5 & 12.92 & 2.04 & 0.65 & 1.99 & 6.07 \\
\hline N4606 .............. & 1 & 12.69 & 2.22 & 0.50 & 2.04 & 6.48 \\
\hline N4630 .............. & 4 & 13.14 & 2.01 & 0.71 & 2.01 & 6.03 \\
\hline N4639 ............. & 3 & 12.19 & 2.24 & 0.68 & 2.37 & 6.85 \\
\hline N4654 ............ & 5 & 11.14 & 2.45 & 0.58 & 2.26 & 7.16 \\
\hline
\end{tabular}


TABLE 7

Coma Cluster E Galaxies

\begin{tabular}{|c|c|c|c|c|c|}
\hline Galaxy & $T$ & $B_{T}$ & $\log D_{25}$ & $\log v_{\mathrm{c}}$ & $-\log w$ \\
\hline N4789 ............... & -5 & 13.12 & 2.06 & 2.67 & 6.79 \\
\hline N4807 .............. & -5 & 14.50 & 1.78 & 2.57 & 6.13 \\
\hline N4872 ............... & -5 & 13.90 & 1.94 & 2.67 & 6.55 \\
\hline N4841A ............. & -5 & 13.77 & 2.01 & 2.66 & 6.68 \\
\hline N4840 .............. & -5 & 14.72 & 1.63 & 2.64 & 5.89 \\
\hline N4841B ............. & -5 & 13.61 & 2.03 & 2.60 & 6.65 \\
\hline N4850 .............. & -5 & 15.22 & 1.60 & 2.49 & 5.69 \\
\hline N4860 .............. & -5 & 14.56 & 1.92 & 2.66 & 6.50 \\
\hline I3957 .............. & -5 & 15.80 & 1.38 & 2.41 & 5.17 \\
\hline I3960 ............... & -5 & 15.90 & 1.38 & 2.48 & 5.24 \\
\hline I3959 .............. & -5 & 15.25 & 1.50 & 2.53 & 5.53 \\
\hline N4864 ............... & -5 & 14.58 & 1.55 & 2.54 & 5.63 \\
\hline N4867 ............... & -5 & 15.44 & 1.60 & 2.58 & 5.78 \\
\hline N4865 .............. & -5 & 14.64 & 1.75 & 2.61 & 6.10 \\
\hline N4869 ............... & -5 & 14.77 & 1.65 & 2.55 & 5.85 \\
\hline N4872a $\ldots \ldots \ldots \ldots \ldots$ & -5 & 15.07 & 1.97 & 2.56 & 6.50 \\
\hline N4874 & -5 & 12.63 & 2.07 & 2.62 & 6.76 \\
\hline N4881 ............... & -5 & 14.59 & 1.77 & 2.56 & 6.10 \\
\hline N4886 $\ldots \ldots \ldots \ldots \ldots$ & -5 & 14.80 & 1.81 & 2.41 & 6.02 \\
\hline N4889 .............. & -5 & 12.53 & 2.24 & 2.84 & 7.32 \\
\hline I $4021 \ldots \ldots \ldots \ldots \ldots \ldots$ & -5 & 15.86 & 1.39 & 2.44 & 5.21 \\
\hline N4895 ............... & -5 & 14.22 & 2.04 & 2.57 & 6.64 \\
\hline N4908 .............. & -5 & 14.66 & 1.80 & 2.55 & 6.15 \\
\hline I $4051 \ldots \ldots \ldots \ldots \ldots . . . . .$. & -5 & 14.17 & 1.78 & 2.59 & 6.14 \\
\hline N4931 ............... & -5 & 14.50 & 2.01 & 2.55 & 6.57 \\
\hline N4957 .............. & -5 & 14.01 & 1.86 & 2.63 & 6.35 \\
\hline
\end{tabular}

${ }^{\text {a }}$ Galaxies showing a large deviation in Fig. $1 b$.

TABLE 8

Coma Cluster S0 GalaXies

\begin{tabular}{|c|c|c|c|c|c|}
\hline Galaxy & $T$ & $B_{T}$ & $\log D_{25}$ & $\log v_{\mathrm{c}}$ & $-\log w$ \\
\hline N4798 ............... & -2 & 14.20 & 1.86 & 2.47 & 6.18 \\
\hline N4816 ................ & -2 & 13.80 & 1.89 & 2.56 & 6.34 \\
\hline N4839 ................ & -2 & 13.02 & 2.38 & 2.66 & 7.42 \\
\hline $\mathrm{Z} 160065 \ldots \ldots \ldots \ldots$ & -2 & 14.10 & 1.80 & 2.51 & 6.11 \\
\hline N4859 ............... & -2 & 14.60 & 1.88 & 2.57 & 6.33 \\
\hline N4871 .............. & -2 & 15.17 & 1.62 & 2.46 & 5.70 \\
\hline N4873 ................ & -2 & 15.07 & 1.69 & 2.41 & 5.79 \\
\hline N4876 ............... & -2 & 15.39 & 1.52 & 2.51 & 5.55 \\
\hline I $4041 \ldots \ldots \ldots \ldots \ldots$ & -2 & 15.30 & 1.60 & 2.33 & 5.53 \\
\hline I $4045 \ldots \ldots \ldots \ldots \ldots$ & -2 & 14.96 & 1.67 & 2.56 & 5.90 \\
\hline N4919 ................ & -2 & 15.08 & 1.81 & 2.45 & 6.07 \\
\hline N4923 ................ & -2 & 14.67 & 1.68 & 2.56 & 5.91 \\
\hline N4926 ................ & -2 & 14.04 & 1.85 & 2.66 & 6.36 \\
\hline N4944 ................ & -2 & 13.80 & 2.02 & 2.54 & 6.57 \\
\hline
\end{tabular}


TABLE 9

Coma Cluster S Galaxies

\begin{tabular}{|c|c|c|c|c|c|c|}
\hline Galaxy & $T$ & $B_{T}$ & $\log D_{25}$ & $R_{25}$ & $\log v_{\mathrm{c}}$ & $-\log w$ \\
\hline I821 & 4 & 14.32 & 1.83 & 0.85 & 2.36 & 6.02 \\
\hline n........... & $\ldots$ & 14.90 & 1.60 & 0.76 & 2.22 & 5.42 \\
\hline $\mathrm{I} 842 \ldots \ldots \ldots \ldots \ldots$ & $\ldots$ & 14.61 & 1.85 & 0.50 & 2.37 & 6.06 \\
\hline I $854 \ldots \ldots \ldots \ldots \ldots$ & $\ldots$ & 14.94 & 1.68 & 0.58 & 2.24 & 5.60 \\
\hline I3913 ............. & $\ldots$ & 15.45 & 1.63 & 0.63 & 2.15 & 5.41 \\
\hline $\mathrm{I} 4210 \ldots \ldots \ldots \ldots$ & $\ldots$ & 15.07 & 1.67 & 0.68 & 2.24 & 5.58 \\
\hline N4735 ............ & $\ldots$ & 15.15 & 1.54 & 0.67 & 2.35 & 5.42 \\
\hline N4921 ............. & 2 & 13.20 & 2.17 & 0.86 & 2.26 & 6.60 \\
\hline N4966 ............. & $\ldots$ & 14.09 & 1.78 & 0.55 & 2.41 & 5.96 \\
\hline N4979 ............ & $\ldots$ & 14.41 & 1.80 & 0.60 & 2.19 & 5.79 \\
\hline N5000 ............. & 4 & 13.78 & 2.01 & 0.77 & 2.17 & 6.19 \\
\hline N5032 $\ldots \ldots \ldots \ldots \ldots$ & 3 & 13.78 & 2.10 & 0.54 & 2.52 & 6.71 \\
\hline N5041 ........... & 6 & 13.98 & 2.00 & 0.79 & 2.39 & 6.39 \\
\hline U7890 ............. & $\ldots$ & 15.00 & 1.60 & 0.71 & 2.31 & 5.51 \\
\hline U7978 .............. & 6 & 14.75 & 1.87 & 0.57 & 2.31 & 6.04 \\
\hline U8229 ............. & 3 & 14.30 & 1.93 & 0.66 & 2.41 & 6.27 \\
\hline U8259 ............. & $\ldots$ & 14.78 & 1.92 & 0.46 & 2.34 & 6.18 \\
\hline Z130006 ............ & $\ldots$ & 14.95 & 1.56 & 0.81 & 2.26 & 5.38 \\
\hline Z130008 ............. & $\ldots$ & 15.06 & 1.41 & 0.73 & 2.24 & 5.05 \\
\hline Z159090 ............ & $\ldots$ & 15.45 & 1.73 & 0.50 & 1.94 & 5.40 \\
\hline Z159101 ............ & $\ldots$ & 15.90 & 1.40 & 0.83 & 2.19 & 4.99 \\
\hline $\mathrm{Z} 160067 \ldots \ldots \ldots \ldots$ & -2 & 15.81 & 1.40 & 0.74 & 2.24 & 5.04 \\
\hline Z160080 ............ & $\ldots$ & 14.84 & 1.72 & 0.79 & 2.03 & 5.47 \\
\hline Z160127 ............ & $\ldots$ & 15.56 & 1.75 & 0.65 & 2.13 & 5.63 \\
\hline Z160139........... & $\ldots$ & 14.97 & 1.76 & 0.53 & 2.10 & 5.62 \\
\hline
\end{tabular}


TABLE 10

E Galaxies in the Virgo, Fornax, Perseus and Coma Clusters in the SAMPLE OF DRESSLER ET AL. 1987

\begin{tabular}{|c|c|c|c|c|c|}
\hline Cluster & Galaxy & $B_{T}$ & $\log D_{25}$ & $\log v_{\mathrm{c}}$ & $-\log w$ \\
\hline \multirow[t]{20}{*}{ Virgo ................ } & N4239 & 13.56 & 2.03 & 1.91 & 5.97 \\
\hline & N4365 & 10.63 & 2.62 & 2.61 & 7.85 \\
\hline & N4374 & 10.15 & 2.59 & 2.68 & 7.85 \\
\hline & N4387 & 12.86 & 2.03 & 2.26 & 6.31 \\
\hline & N4406 & 9.88 & 2.73 & 2.55 & 8.01 \\
\hline & N4434 & 12.80 & 1.93 & 2.21 & 6.06 \\
\hline & N4458 & 12.73 & 2.02 & 2.15 & 6.18 \\
\hline & N4464 & 13.62 & 1.81 & 2.28 & 5.89 \\
\hline & N4472 & 9.51 & 2.79 & 2.67 & 8.25 \\
\hline & N4473 & 11.19 & 2.43 & 2.47 & 7.32 \\
\hline & N4478 & 12.16 & 2.06 & 2.37 & 6.48 \\
\hline & N4486 & 9.57 & 2.70 & 2.73 & 8.12 \\
\hline & N4489 & 12.71 & 2.01 & 1.98 & 5.99 \\
\hline & N4551 & 12.70 & 2.04 & 2.22 & 6.29 \\
\hline & N4552 & 10.87 & 2.49 & 2.59 & 7.56 \\
\hline & N4564 & 11.92 & 2.33 & 2.38 & 7.04 \\
\hline & N4621 & 10.70 & 2.51 & 2.54 & 7.55 \\
\hline & N4636 & 10.22 & 2.56 & 2.50 & 7.62 \\
\hline & N4649 & 9.73 & 2.65 & 2.71 & 8.01 \\
\hline & N4660 & 12.19 & 2.12 & 2.46 & 6.70 \\
\hline \multirow[t]{8}{*}{ Fornax ............. } & N1339 & 12.52 & 2.06 & 2.38 & 6.49 \\
\hline & N1344 & 11.13 & 2.56 & 2.39 & 7.51 \\
\hline & N1374 & 11.85 & 2.17 & 2.41 & 6.75 \\
\hline & N1379 & 11.62 & 2.16 & 2.27 & 6.59 \\
\hline & N1399 & 10.59 & 2.62 & 2.67 & 7.91 \\
\hline & N1404 & 10.90 & 2.30 & 2.58 & 7.18 \\
\hline & N1427 & 11.78 & 2.34 & 2.40 & 7.07 \\
\hline & I2006 & 12.19 & 2.10 & 2.28 & 6.47 \\
\hline \multirow[t]{7}{*}{ Perseus .............. } & N1260 & 13.65 & 1.84 & 2.55 & 6.22 \\
\hline & N1270 & 13.57 & 1.95 & 2.78 & 6.68 \\
\hline & N1272 & 12.23 & 2.09 & 2.67 & 6.85 \\
\hline & N1274 & 14.38 & 1.50 & 2.47 & 5.47 \\
\hline & N1278 & 12.83 & 1.97 & 2.66 & 6.59 \\
\hline & N1282 & 13.36 & 1.93 & 2.58 & 6.44 \\
\hline & $\mathrm{I} 310$ & 13.12 & 1.89 & 2.56 & 6.34 \\
\hline \multirow[t]{18}{*}{ Coma ................ } & N4839 & 13.21 & 2.38 & 2.65 & 7.40 \\
\hline & N4926 & 13.95 & 1.85 & 2.59 & 6.29 \\
\hline & I3959 & 15.07 & 1.50 & 2.48 & 5.48 \\
\hline & I3957 & 15.63 & 1.38 & 2.36 & 5.12 \\
\hline & N4869 & 14.57 & 1.65 & 2.48 & 5.78 \\
\hline & N4876 & 15.22 & 1.52 & 2.44 & 5.48 \\
\hline & $\mathrm{N} 4874^{\mathrm{a}}$ & 12.31 & 2.07 & 2.58 & 6.72 \\
\hline & $\mathrm{N} 4872^{\mathrm{a}}$ & 15.35 & 1.97 & 2.51 & 6.44 \\
\hline & N4867 & 15.28 & 1.60 & 2.54 & 5.73 \\
\hline & $\mathrm{I} 4051$ & 14.01 & 1.78 & 2.56 & 6.11 \\
\hline & N4889 & 12.48 & 2.24 & 2.78 & 7.26 \\
\hline & N4886 & 14.78 & 1.81 & 2.38 & 5.99 \\
\hline & N4864 & 14.62 & 1.55 & 2.47 & 5.57 \\
\hline & I 4045 & 14.96 & 1.67 & 2.52 & 5.85 \\
\hline & I 4021 & 15.62 & 1.39 & 2.39 & 5.16 \\
\hline & N4860 & 14.43 & 1.92 & 2.59 & 6.43 \\
\hline & N4881 & 14.43 & 1.77 & 2.47 & 6.01 \\
\hline & N4841A & 13.51 & 2.01 & 2.58 & 6.60 \\
\hline
\end{tabular}

${ }^{\text {a }}$ Galaxies showing a large deviation in Fig. $1 c$. 


\section{APPENDIX B}

\section{COMPARISON OF DATA SOURCES}

The data used in the present study are adopted from various sources and may be subject to systematic anomalies bound to the individual sources. In order to make the comparisons among the different types of galaxies and among the different clusters of galaxies, we try to find out the formula of transformation among the data from the different sources. The formula of the transformation are derived by the regression-line analyses $(Y=A X+C)$ using the data from $\operatorname{RC} 3\left(B_{T}, \log D_{25}\right.$, $\left.\log W_{20}\right)$ and from Dressler et al. (1987) $\left(\log \sigma_{0}\right)$ as the standard references $(\mathrm{X})$. The resulting coefficients $(A$ and $C)$ are given in Table 11 with the number of sample $(N)$ and the correlation coefficient. The numbers in the sample in Table $11(N)$ are different from those in Tables 1,2 , and $3(n)$, because we use all galaxies available in common to the respective two data sources in question. An outstanding deviation is found for $B_{T}$ in Fukugita et al. (1991), and its transformation to the RC3 system is applied in $\S 2.2$. No other transformations are applied, for they do not affect the essential features of the present analyses.

TABLE 11

Regression Line Analyses $Y=A X+C$ for the Transformation among Different Data Sources

\begin{tabular}{|c|c|c|c|c|c|c|c|}
\hline \multirow[b]{2}{*}{$X$} & \multicolumn{3}{|c|}{$B_{T}$} & \multirow{2}{*}{$\begin{array}{c}\log D_{25} \\
\mathrm{RC} 3\end{array}$} & \multirow{2}{*}{$\begin{array}{c}\log W_{20} \\
\mathrm{RC} 3\end{array}$} & \multicolumn{2}{|c|}{$\log \sigma_{0}$} \\
\hline & $\mathrm{RC} 3$ & $\mathrm{RC} 3$ & $\mathrm{RC} 3$ & & & DLB & DLB \\
\hline$Y$ & BST & DLB & FOT & BST & FOT & DPV & $\mathrm{SGH}$ \\
\hline 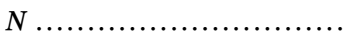 & 93 & 38 & 17 & 99 & 40 & 20 & 18 \\
\hline …........ & 0.9615 & 0.9693 & 0.8003 & 0.8947 & 0.9117 & 0.9006 & 0.9772 \\
\hline$C \ldots \ldots \ldots \ldots \ldots \ldots \ldots \ldots \ldots$ & 0.4685 & 0.3183 & 2.7307 & 0.1680 & 0.2376 & 0.2705 & 0.1069 \\
\hline Correlation Coefficient...... & 0.9870 & 0.9979 & 0.9489 & 0.9769 & 0.9463 & 0.9851 & 0.9827 \\
\hline
\end{tabular}

NoTE.-RC3: de Vaucouleurs et al. 1991; DLB: Dressler et al. 1987; BST: Binggeli et al. 1985; FOT: Fukugita et al. 1991; DPV: Davoust et al. 1985; SGH: Scodeggio et al. 1998a, 1998b.

Bender, R., Burstein, D., \& Faber, S. M. 1992, ApJ, 399, 462

Binggeli, B., Sandage, A., \& Tammann, G. A. 1985, AJ, 90, 1681

Binney, J., \& Tremaine, S. 1987, Galactic Dynamics (Princeton: Princeton Univ. Press)

Brosche, P. 1973, A\&A, 23, 259

Burstein, D., Bender, R., Faber, S. M., \& Nolthenius, R. 1997, AJ, 114, 1365

Chiba, M., \& Yoshii, Y. 1995, ApJ, 442, 82

Davoust, E., Paturel, G., \& Vauglin, I. 1985, A\&AS, 61, 273

de Vaucouleurs, G., de Vaucouleurs, A., Corwin, H. G., Buta, R., Paturel, G., \& Fouqué, P. 1991, Third Reference Catalogue of Bright Galaxies (New York: Springer) (RC3)

Djorgovski, S. 1995, ApJ, 438, L29

Djorgovski, S., \& Davis, M. 1987, ApJ, 313, 59

Dressler, A., Lynden-Bell, D., Burstein, D., Davies, R. L., Faber, S. M., Terlevich, R. J., \& Wegner, G. 1987, ApJ, 313, 42

Faber, S. M., Dressler, A., Davies, R. L., Burstein, D., Lynden-Bell, D., Terlevich, R. J., \& Wegner, G. 1987, in Nearly Normal Galaxies, from the Planck Time to the Present, ed. S. M. Faber (New York: Springer), 175

Faber, S. M., \& Jackson, R. E. 1976, ApJ, 204, 668

Faber, S. M., Wegner, G., Burstein, D., Davies, R. L., Dressler, A., LyndenBell, D., \& Terlevich, R. J. 1989, ApJS, 69, 763

\section{REFERENCES}

Fritsch, Ch., \& Buchert, Th. 1999, A\&A, 344, 749

Fukugita, M., Okamura, S., Tarusawa, K., Rood, H. J., \& Williams, B. A. 1991, ApJ, 376, 8

Hudson, M. J., Lucy, J. R., Smith, R. J. \& Steel, J. 1997, MNRAS, 291, 488

Jerjen, H., \& Tammann, G. A. 1993, A\&A, 276, 1

Koda, J., \& Sofue, Y. 2000, ApJ, submitted

Kodaira, K. 1989, ApJ, 342, 122 Kodaira, K., Okamura, S., \& Ichikawa, S. 1990, Photometric Atlas of Northern Bright Galaxies (Tokyo: Univ. Tokyo Press)

Kodaira, K., Okamura, S., \& Watanabe, M. 1983, ApJ, 274, L49

Kodaira, K., \& Watanabe, M. 1988, AJ, 96, 1593

Kodaira, K., Watanabe, M., \& Okamura, S. 1986, ApJS, 62, 703

Scodeggio, M., Giovanelli, R., \& Haynes, M. P. 1998a, AJ, 116, 2728 .1998b, AJ, 116, 2738

Shimasaku, K. 1993, ApJ, 413, 59

Tully, R. B., \& Fisher, J. R. 1977, A\&A, 54, 661

Watanabe, M., Kodaira, K., \& Okamura, S. 1985, ApJ, 292, 72

Watanabe, M., Yamashita, K., Furuzawa, A., Kunieda, H., Tawara, Y., \& Honda, H. 1999, ApJ, 527, 80

Whitmore, B. C., \& Kirshner, R. P. 1981, ApJ, 250, 43

Yasuda, N., Fukugita, M., \& Okamura, S. 1997, ApJS, 108, 417 